\title{
Opisthorchiidae triad: comparative genomics of the carcinogenic liver flukes using a draft genome of Opisthorchis felineus
}

\author{
N.I. Ershov \\ Institute of Cytology and Genetics SB RAS, Novosibirsk, Russia \\ e-mail: nikotinmail@mail.ru
}

Key words: Opisthorchis felineus, draft genome, comparative genomics, trans-splicing

The liver flukes $O$. felineus, $O$. viverrini and $C$. sinensis are distributed in the vast territories of Asia and Europe and pose a serious threat to the health of the population of endemic areas. Genomics of $O$. felineus have been studied significantly weaker than those of $O$. viverrini and $C$. sinensis. This lack of $O$. felineus genomic data is an obstacle to the development of comparative molecular biological approaches necessary to obtain new knowledge about the biology of these trematodes, to identify essential pathways linked to parasite-host interaction, to predict genes that might contribute to liver fluke pathogenesis. Here we report a draft genome assembly and annotation of $O$. felineus and results of comparative study of genomics and transcriptomics of the three Opisthorchiidae liver flukes. The overall genomic sequences of the three species show relatively high divergence from each other. At the same time, the repertoire of genes and their expression levels (at the whole-body scale) were highly consistent among the opisthorchiids. A comparison of the phylogenetic trees for the three studied opisthorchiids and the data on their synteny suggests that the $O$. viverrini genome was structurally remodeled after it had diverged from its common ancestor with $C$. sinensis. In addition, we have demonstrated that the products of almost half $O$. felineus genes contain the SL sequence, which suggests high importance of trans-splicing in the regulation of RNA processing in liver flukes. The study is also focused on some gene networks involved in regulation of host-parasite interaction. Availability of a genome for $O$. felineus should help support the development of comparative genomics, proteomics and other -omics studies necessary for understanding the parasites evolution and development of novel drugs and vaccines against liver flukes. This work was partly supported by the Russian Scientific Foundation (grant No. 18-74-00101). 